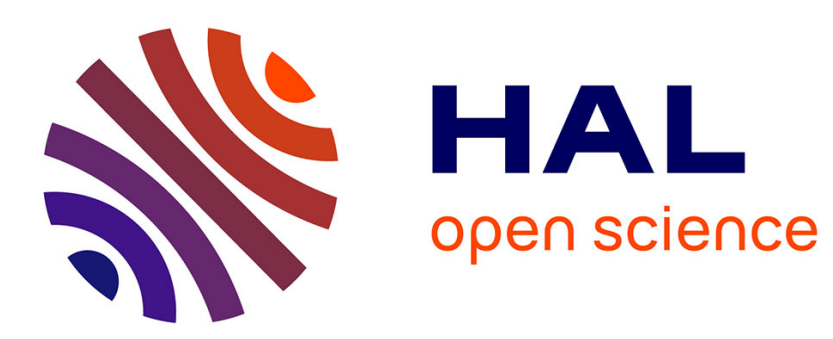

\title{
Starting-point bias and respondent uncertainty in dichotomous choice contingent valuation surveys
}

Emmanuel Flachaire, Guillaume Hollard

\section{To cite this version:}

Emmanuel Flachaire, Guillaume Hollard. Starting-point bias and respondent uncertainty in dichotomous choice contingent valuation surveys. Resource and Energy Economics, 2007, 29 (3), pp.183-194. 10.1016/j.reseneeco.2007.05.003 . halshs-00175925

\section{HAL Id: halshs-00175925 \\ https://shs.hal.science/halshs-00175925}

Submitted on 1 Oct 2007

HAL is a multi-disciplinary open access archive for the deposit and dissemination of scientific research documents, whether they are published or not. The documents may come from teaching and research institutions in France or abroad, or from public or private research centers.
L'archive ouverte pluridisciplinaire HAL, est destinée au dépôt et à la diffusion de documents scientifiques de niveau recherche, publiés ou non, émanant des établissements d'enseignement et de recherche français ou étrangers, des laboratoires publics ou privés. 


\title{
StaRTing-POINT Bias And RESPONDENT Uncertainty in Dichotomous Choice Contingent Valuation Surveys
}

\author{
by \\ Emmanuel Flachaire \\ Université Paris 1 Panthéon-Sorbonne \\ and \\ Guillaume Hollard \\ Université de Marne la Vallée
}

\begin{abstract}
May 2007
In this article, we develop a dichotomous choice model with follow-up questions that describes the willingness to pay being uncertain in an interval. The initial response is subject to starting point bias. Our model provides an alternative interpretation of the starting point bias in the dichotomous choice valuation surveys. Using the Exxon Valdez survey, we show that, when uncertain, individuals tend to answer "yes".
\end{abstract}

Key words: starting point bias, preference uncertainty, contingent valuation JEL Classification: Q26, C81

We are very grateful to Brett Day, Nick Hanley, Bengt Kriström and Jason Shogren for their helpful comments and suggestions. We are grateful to numerous seminar participants at the Universities of Evry, Kyoto, Lille, Montpellier and Stirling. 


\section{Introduction}

The NOAA panel recommends the use of a dichotomous choice format in contingent valuation (CV) surveys. This format has several advantages: it is incentive-compatible, simple and cognitively manageable. Furthermore, respondents face a familiar task, similar to real referenda. The use of a single valuation question, however, presents the inconvenience of providing the researcher with only limited information. To gather more information, Hanemann et al. (1991) proposed adding a follow-up question. This is the double-bounded model. This format, however, has been proved to be sensitive to starting point bias, that is, respondents anchor their willingness-to-pay (WTP) to the bids. It implies that WTP estimates may vary as a function of the bids. Many authors propose some specific models to handle this problem (Herriges and Shogren 1996, Alberini et al. 1997, DeShazo 2002, Whitehead 2002, Cooper et al. 2002, Flachaire and Hollard 2006). The behavioral assumption behind these models is that respondents hold a unique and precise willingness-to-pay prior to the survey. Observed biases are interpreted as a distortion of this initial willingness-to-pay during the survey.

Independently, several studies document the fact that individuals are rather unsure of their own willingness-to-pay (Li and Mattsson 1995, Ready et al. 1995, 2001, Welsh and Poe 1998, van Kooten et al. 2001, Hanley and Kriström 2002, Alberini et al. 2003). To account for such uncertainty, these studies allow respondents to use additional answers to valuation questions. Rather than the usual "yes", "no" and "don't know" alternatives, intermediate responses, such as "probably yes" or "probably no", are allowed. Alternatively, an additional question asks respondents how certain they are of their answers and provides a graduated scale.

In contingent valuation, starting-point bias and respondent's uncertainty have been handled in separate studies. In this article we develop a dichotomous choice model hereafter called the Range model - in which individuals hold a range of acceptable values, rather than a precisely defined value of their willingness-to-pay. The range model is drawn from the principle of coherent arbitrariness, suggested by Ariely et al. (2003b). Prior to the survey, the true willingness to pay is assumed to be uncertain in an interval with upper and lower bounds. Confronted with the first valuation question, respondents select a value and then act on the basis of that selected value. Because of this initial uncertainty, the initial choice is subject to starting point bias. In contrast, the subsequent choices are no longer sensitive to the bid offers. A clear-cut prediction follows: biases occur within a given range and affect the first answer only. The Range model thus provides an alternative interpretation of the starting point bias in the dichotomous choice valuation surveys.

An empirical study is presented to compare various models, using the well-known Exxon Valdez contingent valuation survey. Results show that a special case of the proposed Range model, in which a "yes" response is given when the bid value falls within the range of acceptable values, is supported by the data, i.e. when uncertain, individuals tend to say "yes". 
The article is organized as follows. The following section presents the Range model and the respondent's decision process. The subsequent sections provide estimation details, give further interpretation and present an application. Conclusions appear in the final section.

\section{The Range model}

The Range model derives from the principle of "coherent arbitrariness" (Ariely et al. 2003b). These authors conducted a series of valuation experiments (i.e. experiments in which the subjects have to set values for objects they are not familiar with). They observed that "preferences are initially malleable but become imprinted (i.e. precisely defined and largely invariant) after the individual is called upon to make an initial decision". But, prior to imprinting, preferences are "arbitrary, meaning that they are highly responsive to both positive and normative influences".

In a double-bounded CV survey, two questions are presented to respondents. The first question is "Would you agree to pay $\mathrm{x} \$ ? "$. The second, or follow-up, question is similar but asks for a higher bid offer if the initial answer is yes and a lower bid offer otherwise. Confronted with these iterative questions, with two successive bids proposed, the principle of coherent arbitrariness leads us to consider a three-step decision process:

1. Prior to a valuation question, the respondent holds a range of acceptable values

2. Confronted with a first valuation question, the respondent selects a value inside that range

3. The respondent answers the questions according to the selected value.

The following subsections detail each step.

\subsection{A range of acceptable values}

At first, let us assume that a respondent $i$ does not hold a precise willingness-to-pay but rather an interval of acceptable values:

$$
w t p_{i} \in\left[\underline{W}_{i}, \bar{W}_{i}\right] \quad \text { with } \quad \bar{W}_{i}-\underline{W}_{i}=\delta .
$$

The lower bound and the upper bound are different for each respondent, but we assume the width of the range $\delta$ to be constant across individuals. ${ }^{1}$

\footnotetext{
${ }^{1}$ It would be interesting to consider a model in which $\delta$ varies across individuals. Some variables that are proved to play a role in individual value assessment (such as repeated exposure to the good or representation of the good (Flachaire and Hollard 2007)) may also influence the length of the range. This requires a particular treatment which is beyond the scope of this paper.
} 
Several psychological and economic applications support this idea. For instance, Tversky and Kahneman (1974) and Ariely et al. (2003b, 2003a) suggest the existence of such an interval. In addition, several studies in contingent valuation explore response formats that allow for the expression of uncertainty, among others see Li and Mattsson (1995), Ready et al. (1995), Welsh and Poe (1998), van Kooten et al. (2001), Hanley and Kriström (2002) and Alberini et al. (2003). These studies also conclude that there is a range of values for which respondents are uncertain.

\subsection{Selection of a particular value}

Confronted with a first bid offer $b_{1 i}$ a respondent $i$ selects a specific value inside his range of acceptable values $\left[\underline{W}_{i}, \bar{W}_{i}\right]$. The selection rule can take different forms. We propose a selection rule in which the respondent selects a value so as to minimize the distance between his range of willingness-to-pay and the proposed bid:

$$
W_{i}=\operatorname{Min}_{w t p_{i}}\left|w t p_{i}-b_{1 i}\right| \quad \text { with } \quad w t p_{i} \in\left[\underline{W}_{i}, \bar{W}_{i}\right] .
$$

This selection rule has attractive features. It is very simple and tractable. It is also in accordance with the literature on anchoring, which states that the proposed bid induces subject to revise their willingness to pay as if the proposed bid conveyed some information about the "right" value (Chapman and Johnson 1999). At a more general level, the literature on cognitive dissonance suggests that subjects act so as to minimize the gap between their own opinion and the one conveyed by new information.

In this range model the first bid plays the role of an anchor: it attracts the willingnessto-pay. A different $b_{1 i}$ results in the selection of a different value $W_{i}$. Thus, this selection rule should exhibit a sensitivity of the first answer to the first bid, that is, an anchoring effect. Consequently, it is expected to produce anomalies such as starting point bias.

\subsection{Answers to questions}

The last step of the decision process deals with the respondent's answer to questions. It is straightforward that a respondent will answer yes if the bid is less than the lower bound of his range of acceptable value $\underline{W}_{i}$. And he will answer no if the bid is higher than the upper bound of his range $\bar{W}_{i}$. However, it is less clear what is happening when the bid belongs to the interval of acceptable values.

\section{- Answers to the first question -}

A respondent $i$ will agree to pay any amount below $\underline{W}_{i}$ and refuse to pay any amount that exceeds $\bar{W}_{i}$. When the first bid belongs to his interval of acceptable values, he may accept or refuse the bid offer. Here, we do not impose a precise rule: respondents 
can answer yes or no with any probability when the bid offer belongs to the interval. If the bid belongs to the range of acceptable values, respondents answer yes to the first question with a probability $\xi$ and no with a probability $1-\xi$. Thus, the probability that a respondent $i$ will answer yes to the first question is equal to: ${ }^{2}$

$$
P(\text { yes })=P\left(b_{1 i}<\underline{W}_{i}\right)+\xi P\left(\underline{W}_{i}<b_{1 i}<\bar{W}_{i}\right) \quad \text { with } \quad \xi \in[0,1] .
$$

In other words, a respondent's first answer is yes with a probability 1 if the bid is below his range of acceptable values and with a probability $\xi$ if the bid belongs to his range. A $\xi$ close enough to 1 (resp. 0) means that the respondent tends to answer yes (resp. no) when the bid belongs to the range of acceptable values. Estimation of the model will provide an estimate of $\xi$.

\section{- Answers to follow-up questions -}

The uncertainty that arises in the first answer disappears in the follow-up answers. A respondent answers yes to the follow-up question if the bid $b_{2 i}$ is below his willingness-topay, $W_{i}>b_{2 i}$; and $n o$ if the bid is above his willingness-to-pay, $W_{i}<b_{2 i}$ (by definition, the follow-up bid is higher or smaller than the first bid, that is $b_{2 i} \neq b_{1 i}$ ).

\section{Estimation}

In this section, we present in detail how to estimate the Range model. It is assumed that if the first bid $b_{1 i}$ belongs to the interval of acceptable values of respondent $i,\left[\underline{W}_{i} ; \bar{W}_{i}\right]$, he will answer yes with a probability $\xi$ and no with a probability $1-\xi$. We can write these two probabilities as follows:

$$
\xi=\frac{P\left(\underline{W}_{i}<b_{1 i}<W_{i}^{\xi}\right)}{P\left(\underline{W}_{i}<b_{1 i}<\bar{W}_{i}\right)} \quad \text { and } \quad 1-\xi=\frac{P\left(W_{i}^{\xi}<b_{1 i}<\bar{W}_{i}\right)}{P\left(\underline{W}_{i}<b_{1 i}<\bar{W}_{i}\right)},
$$

with $W_{i}^{\xi} \in\left[\underline{W}_{i} ; \bar{W}_{i}\right]$. Note that, when $\xi=0$ we have $W_{i}^{\xi}=\underline{W}_{i}$, and when $\xi=1$ we have $W_{i}^{\xi}=\bar{W}_{i}$. From (4) and (3), the respondent $i$ answers yes or no to the first question with the following probabilities

$$
P(\text { yes })=P\left(W_{i}^{\xi}>b_{1 i}\right) \quad \text { and } \quad P(n o)=P\left(W_{i}^{\xi} \leq b_{1 i}\right) .
$$

It is worth noting that these probabilities are similar to the probabilities derived from a single-bounded model with $W_{i}^{\xi}$ assumed to be the willingness-to-pay of respondent $i$. It follows that the mean value of WTPs obtained with a single-bounded model would correspond to the mean of the $W_{i}^{\xi}$ in our model, for $i=1, \ldots, n$. The use of follow-up questions will lead us to identify and estimate $\xi$ and to provide a range of values rather than a single mean of WTPs.

\footnotetext{
${ }^{2} P($ yes $)=P\left(\right.$ yes $\left.\mid b_{1 i}<\underline{W}_{i}\right) P\left(b_{1 i}<\underline{W}_{i}\right)+P\left(\right.$ yes $\left.\mid \underline{W}_{i}<b_{1 i}<\bar{W}_{i}\right) P\left(\underline{W}_{i}<b_{1 i}<\bar{W}_{i}\right)+P\left(\right.$ yes $\mid b_{1 i}>$ $\left.\bar{W}_{i}\right) P\left(b_{1 i}>\bar{W}_{i}\right)$ where the conditional probabilities are respectively equal to $1, \xi$ and 0 .
} 
If the initial bid belongs to his range of acceptable values, respondent $i$ selects the value $W_{i}=b_{1 i}$, see (2). If his first answer is yes, a follow-up higher bid $b_{2 i}^{h}>b_{1 i}$ is proposed and his second answer is necessarily no, because $W_{i}<b_{2 i}^{h}$. Conversely, if his first answer is no, a follow-up lower bid $b_{2 i}^{l}<b_{1 i}$ is proposed and his second answer is necessarily yes, because $W_{i}>b_{2 i}^{l}$. It follows that, if the first and the second answers are similar, the first bid is necessarily outside the interval $\left[\underline{W}_{i} ; \bar{W}_{i}\right]$ and the probabilities of answering no-no and yes-yes are respectively equal to

$$
P(n o, n o)=P\left(\bar{W}_{i}<b_{2 i}^{l}\right) \quad \text { and } \quad P(\text { yes, yes })=P\left(\underline{W}_{i}>b_{2 i}^{h}\right) .
$$

If the answers to the initial and the follow-up questions are respectively yes and no, two cases are possible: the first bid is below the range of acceptable values and the second bid is higher than the selected value $W_{i}=\underline{W}_{i}$, otherwise the first bid belongs to the range of values. We have

$$
\begin{aligned}
P(\text { yes, no }) & =P\left(b_{1 i}<\underline{W}_{i}<b_{2 i}^{h}\right)+\xi P\left(\underline{W}_{i}<b_{1 i}<\bar{W}_{i}\right) \\
& =P\left(b_{1 i}<\underline{W}_{i}<b_{2 i}^{h}\right)+P\left(\underline{W}_{i}<b_{1 i}<W_{i}^{\xi}\right) \\
& =P\left(\underline{W}_{i}<b_{2 i}^{h}\right)-P\left(W_{i}^{\xi}<b_{1 i}\right) .
\end{aligned}
$$

Similarly, the probability that respondent $i$ will answer successively no and yes is:

$$
\begin{aligned}
P(\text { no, yes }) & =P\left(b_{2 i}^{l}<\bar{W}_{i}<b_{1 i}\right)+(1-\xi) P\left(\underline{W}_{i}<b_{1 i}<\bar{W}_{i}\right) \\
& =P\left(W_{i}^{\xi}<b_{1 i}\right)-P\left(\bar{W}_{i}<b_{2 i}^{l}\right) .
\end{aligned}
$$

To make the estimation possible, a solution would be to rewrite all the probabilities in terms of $W_{i}^{\xi}$. In our model, we assume that the range of acceptable values has a width which is the same for all respondents. It allows us to define two parameters:

$$
\delta_{1}=\underline{W}_{i}-W_{i}^{\xi} \quad \text { and } \quad \delta_{2}=\bar{W}_{i}-W_{i}^{\xi} .
$$

Note that $\delta_{1} \leq 0$ and $\delta_{2} \geq 0$ because $W_{i}^{\xi} \in\left[\underline{W}_{i} ; \bar{W}_{i}\right]$. Using (12) in (6), (9) and (11), we have

$$
\begin{aligned}
P(\text { no, no }) & =P\left(W_{i}^{\xi}<b_{2 i}^{l}-\delta_{2}\right), & P(\text { no, yes }) & =P\left(b_{2 i}^{l}-\delta_{2}<W_{i}^{\xi}<b_{1 i}\right), \\
P(\text { yes }, \text { yes }) & =P\left(W_{i}^{\xi}>b_{2 i}^{h}-\delta_{1}\right), & P(\text { yes }, \text { no }) & =P\left(b_{1 i}<W_{i}^{\xi}<b_{2 i}^{h}-\delta_{1}\right) .
\end{aligned}
$$

Let us consider that the willingness-to-pay is defined as,

$$
W_{i}^{\xi}=\alpha+X_{i} \beta+u_{i}, \quad u_{i} \sim N\left(0, \sigma^{2}\right),
$$

where the unknown parameters $\beta, \alpha$ and $\sigma^{2}$ are respectively a $k \times 1$ vector and two scalars, $X_{i}$ is a $1 \times k$ vector of explanatory variables. The number of observations is equal to $n$ and the error term $u_{i}$ is Normally distributed with a mean of zero and a variance of $\sigma^{2}$. This model can easily be estimated by maximum likelihood, using the log-likelihood function

$$
\begin{aligned}
l(y, \beta)= & \sum_{i=1}^{n}\left(r_{1 i} r_{2 i} \log [\text { P(yes,yes })\right]+r_{1 i}\left(1-r_{2 i}\right) \log [P(\text { yes }, \text { no })] \\
& \left.\quad+\left(1-r_{1 i}\right) r_{2 i} \log [P(\text { no }, \text { yes })]+\left(1-r_{1 i}\right)\left(1-r_{2 i}\right) \log [P(\text { no }, \text { no })]\right),
\end{aligned}
$$


where $r_{1}$ (resp. $r_{2}$ ) is a dummy variable which is equal to 1 if the answer to the first bid (resp. to the second) is yes, and is equal to 0 if the answer is no. To estimate our model, we can derive from (13) and (14) the probabilities that should be used:

$$
\begin{aligned}
& P(\text { no }, \text { no })=\Phi\left[\left(b_{2 i}^{l}-\delta_{2}-\alpha-X_{i} \beta\right) / \sigma\right], \\
& P(\text { no, yes })=\Phi\left[\left(b_{1 i}-\alpha-X_{i} \beta\right) / \sigma\right]-\Phi\left[\left(b_{2 i}^{l}-\delta_{2}-\alpha-X_{i} \beta\right) / \sigma\right], \\
& P(\text { yes, no })=\Phi\left[\left(b_{2 i}^{h}-\delta_{1}-\alpha-X_{i} \beta\right) / \sigma\right]-\Phi\left[\left(b_{1 i}-\alpha-X_{i} \beta\right) / \sigma\right], \\
& P(\text { yes, yes })=1-\Phi\left[\left(b_{2 i}^{h}-\delta_{1}-\alpha-X_{i} \beta\right) / \sigma\right] .
\end{aligned}
$$

Non-negativity of the probabilities (18) and (19) require respectively $b_{1 i}>b_{2 i}^{l}-\delta_{2}$ and $b_{2 i}^{h}+\delta_{1}>b_{1 i}$. We have defined $\delta_{1} \leq 0$ and $\delta_{2} \geq 0$, see (12): in such cases the probabilities (18) and (19) are necessarily positive. However, the restrictions $\delta_{1} \leq 0$ and $\delta_{2} \geq 0$ are not automatically satisfied in the estimation. To overcome this problem, we can consider a more general model, for which our Range model becomes a special case.

\subsection{Interrelation with the Shift model}

It is worth noting that the probabilities (13) and (14) are quite similar to the probabilities derived from a Shift model (Alberini et al. 1997), but in which we consider two different shifts. Indeed, in a Shift model, respondents are supposed to answer the first question with a prior willingness-to-pay $W_{i}$ and the second question with an updated willingnessto-pay defined as:

$$
W_{i}^{\prime}=W_{i}+\delta
$$

The probability of answering successively yes and no is:

$$
P(\text { yes, no })=P\left(b_{1 i}<W_{i} \cap W_{i}^{\prime}<b_{2 i}^{h}\right)=P\left(b_{1 i}<W_{i}<b_{2 i}^{h}-\delta\right),
$$

which is equal to the corresponding probability in (14) with $\delta=\delta_{1}$. Similar calculations can be made for the other probabilities, to show that the Range model can be estimated as a model with two different shifts in ascending/descending sequences. The underlying decision process is very different from the one developed in the Range model. In the Shift model, respondents answer questions according to two different values of WTP, $W_{i}$ and $W_{i}^{\prime}$. The first bid offer is interpreted as providing information about the cost or the quality of the object. Indeed, a respondent can interpret a higher bid offer as paying more for the same object and a lower bid offer as paying less for a lower quality object. Alternatively, a higher bid can make no sense to the individual, if delivery was promised at the lower bid.

\subsection{Random-effect model}

Cameron and Quiggin (1994) propose taking into account the dynamic aspect of followup questions: they suggest specification allowing the initial and follow-up answers to be 
based on two different WTP values. The WTP is broken down in two parts, a fixed component and a varying component over repeated questions. The random effect model can be written:

$$
\left\{\begin{array}{l}
W_{1 i}=W_{i}^{\star}+\varepsilon_{1 i} \\
W_{2 i}=W_{i}^{\star}+\varepsilon_{2 i}
\end{array} \quad \text { where } \quad W_{i}^{\star}=\alpha+X_{i} \beta+\nu_{i} .\right.
$$

The difference between the two WTP values is due to the random shocks $\varepsilon_{1 i}$ and $\varepsilon_{2 i}$, assumed to be independent. The fixed component $W_{i}^{\star}$ can be split into two parts. $X_{i} \beta$ represent the part of the willingness-to-pay due to observed individual specific characteristics. $\nu_{i}$ varies with the individual, but remains fixed over the indivual's responses: it relates unobserved individual heterogeneity and introduces a correlation between $W_{1 i}$ and $W_{2 i}$. The correlation is high (resp. low) if the variance of the fixed component is large (resp. small) relative to the variance of the varying component, see Alberini et al. (1997) for more details. At the limit, if the two WTP values are identical, $W_{1 i}=W_{2 i}$, the correlation coefficient is equal to one, $\rho=1$.

Alberini et al. (1997) have modified this random-effect model to the case of the Shift model. Since the Range model can be estimated as a model with two different shifts in ascending/descending sequences (see above), the use of a random-effect model in the case of the Range model is straightforward. From equations (17), (18), (19) and (20), we can write the probability that the individual $i$ answers yes to the $j^{\text {th }}$ question, $j=1,2$ :

$$
P\left(W_{j i}>b_{j i}\right)=\Phi\left[\left(\alpha+X_{i} \beta-b_{j i}+\delta_{1} D_{j} r_{1 i}+\delta_{2} D_{j}\left(1-r_{1 i}\right)\right) / \sigma\right],
$$

where $D_{1}=0, D_{2}=1$, and $r_{1 i}$ equals 1 if the answer to the first question is yes and 0 otherwise. Consequently, the Range model can be estimated from the following bivariate probit model:

$$
P(\text { yes, yes })=\Phi\left[\alpha_{1}+X_{i} \theta+\gamma b_{1 i} ; \alpha_{2}+X_{i} \theta+\gamma b_{2 i}+\lambda r_{1 i} ; \rho\right]
$$

The parameters are interrelated according to:

$$
\alpha=-\alpha_{1} / \gamma, \quad \beta=-\theta / \gamma, \quad \sigma=-1 / \gamma, \quad \delta_{1}=-\lambda / \gamma \quad \text { and } \quad \delta_{2}=\left(\alpha_{1}-\alpha_{2}\right) / \gamma .
$$

Estimation with a bivariate probit model based on equation (25) does not impose any restriction on the parameters. The Range model is obtained if $\delta_{1} \leq 0$ and $\delta_{2} \geq 0$; the Shift model is obtained if $\delta_{1}=\delta_{2}$. It is clear that the Range model and the Shift model are non-nested; they can be tested through (25).

\section{Interpretation}

We have seen above that the estimation of the Range model derives from a general model, that also encompasses the Shift model proposed by Alberini et al. (1997), see (25) and (26). Estimation of model (15), based on equation (25), provides estimates of 
$\alpha, \beta, \sigma, \delta_{1}$ and $\delta_{2}$, from which we can estimate a mean $\mu_{\xi}$ and a dispersion $\sigma$ of the willingness-to-pay - (Hanemann and Kanninen 1999) by

$$
\mu_{\xi}=n^{-1} \sum_{i=1}^{n} W_{i}^{\xi}=n^{-1} \sum_{i=1}^{n}\left(\alpha+X_{i} \beta\right)
$$

This last mean of WTPs would be similar to the mean of WTPs estimated using the first questions only, that is, based on the single-bounded model.

Additional information can be obtained from the use of follow-up questions: estimates of $\delta_{1}$ and $\delta_{2}$ allows us to estimate a range of means of WTPs. The mean value of WTPs estimated from our model $\mu^{\xi}$ is the mean of the estimates of $W_{i}^{\xi}$ for all the respondents, $i=1, \ldots, n$. From (12), we can derive the lower bounds of the range of acceptable values for all respondents and a mean of WTPs associated with it:

$$
\mu_{0}=n^{-1} \sum_{i=1}^{n} \underline{W}_{i}=n^{-1} \sum_{i=1}^{n}\left(W_{i}^{\xi}+\delta_{1}\right)=\mu_{\xi}+\delta_{1}, \quad \delta_{1} \leq 0 .
$$

It would be the mean of WTPs when respondents always answer no if the bid belongs to their range of acceptable value. Similarly, we can derive the upper bounds of their range,

$$
\mu_{1}=n^{-1} \sum_{i=1}^{n} \bar{W}_{i}=n^{-1} \sum_{i=1}^{n}\left(W_{i}^{\xi}+\delta_{2}\right)=\mu_{\xi}+\delta_{2}, \quad \delta_{2} \geq 0 .
$$

It follows that we can provide a range of means of WTPs

$$
\left[\mu_{0} ; \mu_{1}\right]=\left[\mu_{\xi}+\delta_{1} ; \mu_{\xi}+\delta_{2}\right] \quad \text { with } \quad \delta_{1} \leq 0, \quad \text { and } \quad \delta_{2} \geq 0
$$

This range can be estimated with $\hat{\mu}_{\xi}, \hat{\delta}_{1}$ and $\hat{\delta}_{2}$. The lower bound $\mu_{0}$ corresponds to the case where respondents always answer no if the bid belongs to the range of acceptable values $(\xi=0)$. Conversely, the upper bound $\mu_{1}$ corresponds to the case where respondents always answer yes if the bid belongs to the range of acceptable values $(\xi=1)$. How respondents answer the question when the bid belongs to the range of acceptable values can be tested as follows:

- respondents always answer no corresponds to the null hypothesis $H_{0}: \delta_{1}=0$,

- respondents always answer yes corresponds to the null hypothesis $H_{0}: \delta_{2}=0$.

Finally, an estimation of the probability $\xi$ would be useful. For instance, we could conclude that when the first bid belongs to the range of acceptable values, respondents answer yes in $(100 \hat{\xi}) \%$ of cases. If the first bids are drawn randomly from a probability distribution, $\xi$ can be rewritten

$$
\xi=\frac{P\left(\mu_{0}<b_{1 i}<\mu_{\xi}\right)}{P\left(\mu_{0}<b_{1 i}<\mu_{1}\right)} .
$$

In addition, if the set of first bids are drawn from a uniform distribution by the surveyors, it can be estimated by $\hat{\xi}=\hat{\delta}_{1} /\left(\hat{\delta}_{1}-\hat{\delta}_{2}\right)$. 


\section{Application}

Since independent variables other than the bid are not needed to estimate the Range model, we can use data from previously published papers on this topic. In this application, we use data from the well-known Exxon Valdez contingent valuation survey. ${ }^{3}$ The willingness-to-pay question asked how the respondent would vote on a plan to prevent another oil spill similar in magnitude to the Exxon Valdez spill. Details about the Exxon Valdez oil spill and the contingent valuation survey can be found in Carson et al. (2003)

\section{$5.1 \quad$ Results}

With the assumption that the distribution of WTP is lognormal, results in Alberini et al. show evidence of a downward shift. Here, we consider the more general model given in (25) from which the Double-bounded, Shift and Range models are special cases.

Estimation results are given in Table 1. We use the same model as in Alberini et al.: there are no covariates and the distribution of the WTP is assumed lognormal $(\theta=0$ and $b_{i j}$ are replaced by $\log b_{i j}$ in (25)). The mean of $\log$ WTP is given by $\alpha=-\alpha_{1} \gamma$ and the median of WTP is given by $\exp (\alpha)$. Estimation results of the Single-bounded model are obtained from a probit model. Estimation results obtained from a bivariate probit model with no restrictions in (25) are presented in column $\mathcal{M}$; the Double-bounded model is obtained with $\delta_{1}=\delta_{2}=0$; the Shift model is obtained with $\delta_{1}=\delta_{2}$ and the Range $_{\text {yes }}$ model is obtained with $\delta_{2}=0$.

Table 1: Exxon Valdez Oil Spill Survey: Random-effect models

\begin{tabular}{cccccc}
\hline Parameter & Single & $\begin{array}{c}\text { Double } \\
\left(\delta_{1}=\delta_{2}=0\right)\end{array}$ & $\begin{array}{c}\text { Shift } \\
\left(\delta_{1}=\delta_{2}\right)\end{array}$ & $\begin{array}{c}\mathcal{M} \\
(\text { n.c. })\end{array}$ & $\begin{array}{c}\text { Range }_{\text {yes }} \\
\left(\delta_{2}=0\right)\end{array}$ \\
\hline \hline$\alpha$ & 3.727 & 3.080 & 3.754 & 3.797 & 3.789 \\
& $(0.124)$ & $(0.145)$ & $(0.127)$ & $(0.129)$ & $(0.134)$ \\
$\sigma$ & 3.149 & 3.594 & 3.236 & 3.298 & 3.459 \\
& $(0.432)$ & $(0.493)$ & $(0.421)$ & $(0.387)$ & $(0.272)$ \\
$\delta_{1}$ & & & -1.108 & -1.424 & -1.583 \\
& & & $(0.212)$ & $(0.356)$ & $(0.222)$ \\
$\delta_{2}$ & & & & -0.062 & \\
& & & & $(0.114)$ & \\
$\rho$ & & 0.694 & 0.770 & 0.997 & 0.998 \\
$\ell$ & -695.51 & -1345.70 & -1303.36 & -1301.32 & -1301.45 \\
\hline
\end{tabular}

Note: standard errors are in italics; n.c.: no constraints.

From Table 1, we can see that the estimates of the mean of log WTP in the Singlebounded and Double-bounded models are very different (3.727 vs. 3.080). Such incon-

\footnotetext{
${ }^{3}$ The bid values are given in Alberini, Kanninen, and Carson (1997, Table 1).
} 
sistent results lead us to consider the Shift model to control for such effects. It is clear that the estimates of the mean of log WTP in the Single-bounded model and in the Shift model are very close (3.727 vs. 3.754), and that the Double-bounded model does not fit the data as well as the Shift model. Indeed, we reject the null hypothesis $\delta_{1}=0$ from a likelihood-ratio test $(L R=84.68$ and $P<0.0001) .{ }^{4}$ To go further, we consider estimation results obtained from the model defined in (25) with no restrictions (column $\mathcal{M})$. On the one hand, we reject the null hypothesis $\delta_{1}=\delta_{2}$ from a likelihood-ratio test $(L R=4.08$ and $P=0.043)$. It suggests that the Shift model does not fit the data as well as model $\mathcal{M}$. On the another hand, we cannot reject the null hypothesis $\delta_{2}=0$ $(L R=0.26$ and $P=0.61)$. It leads us to select the Range yes model hereafter.

The estimated values of the parameters $\delta_{1}$ and $\delta_{2}$ allow us to interpret the model as a Range model $\left(\delta_{1} \leq 0, \delta_{2}=0\right)$. Respondents are unsure of their willingness-to-pay in an interval; they answer yes if the initial bid offer belongs to their interval of acceptable values. We compute an interval of the median WTP:

$$
\left[\exp \left(\hat{\alpha}-\hat{\delta}_{1}\right) ; \exp \left(\hat{\alpha}-\hat{\delta}_{2}\right)\right]=[9.45 ; 44.21]
$$

This interval suggests that, if the respondents answer no if the initial bid belongs to their range of acceptable values, the median WTP is equal to 9.45; if the respondents answer yes if the initial bid belongs to their range of acceptable values, the median WTP is equal to 44.21 (see Section 4).

\subsection{Main findings}

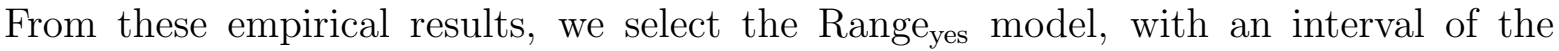
median WTP $[9.45 ; 44.21]$. Previous researchers have also found that, when uncertain, individuals tend to say yes (Ready et al. 2001). New with the Range model is the fact that no additional question such as "how certain are you of your answer?" is required. From our results, several conclusions can be drawn:

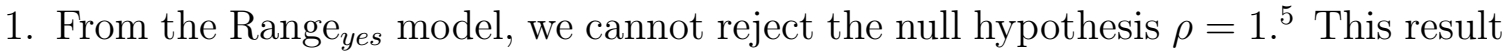
has an important implication. It suggests that the underlying decision process defined in the Range model is supported by the data. Confronted with an initial bid, respondents select a value, then they answer both the first and the second questions according to the same value (see Sections 2 and 3.2). This is in sharp contrast to the existing literature that explains anomalies by the fact that respondents use two different values to answer the first and follow-up questions. ${ }^{6}$ The

\footnotetext{
${ }^{4} \mathrm{~A}$ LR test is equal to twice the difference between the maximized value of the loglikelihood functions (given in the last line $\ell$ ); it is asymptotically distributed as a Chi-squared distribution.

${ }^{5}$ Estimation results of the Range and of the Range yes $_{\text {models obtained by using the constraint } \rho=1}$ are not reported: they are similar to those obtained without imposing this constraint and the estimates of the loglikelihood functions are identical.

${ }^{6}$ Cameron and Quiggin 1994, Kanninen 1995, Herriges and Shogren 1996, Alberini et al. 1997, Whitehead 2002.
} 
Range model supports the view that anomalies can be explained by a specific respondent's behavior prior to the first question, rather than by a change between the first and the second questions.

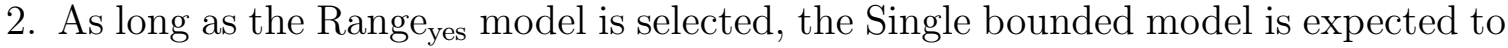
elicit the upper bound of the individual's range of acceptable WTP values. Indeed, in the case of Exxon Valdez, the estimated median WTP is equal to $\exp (\hat{\alpha})=41.55$. This value is very close to the upper bound provided by the interval of the median

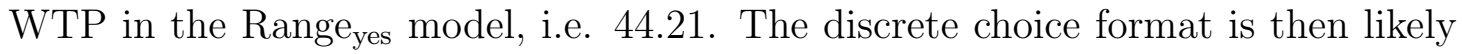
to overestimate means or medians compared to other surveys' formats. It confirms previous research showing that, with the same underlying assumptions, the discrete choice format leads to a systematically higher estimated mean WTP than the openended format (Green et al. 1998) or the payment card format (Ready et al. 2001).

3. Existing results suggest that anomalies occur in ascending sequences only (i.e. after a yes to the initial bid). ${ }^{7}$ DeShazo (2002) offers a prospect-theory explanation, interpreting the first bid as playing the role of a reference point. The Range model offers an alternative explanation: anomalies come from the fact that, when uncertain, respondents tend to answer yes. Indeed, if the bid belongs to his range of acceptable values, a respondent answers yes to the first question and necessarily no to the second question (see Section 2). This specific behavior occurs in ascending sequences only. Such asymmetry can be viewed from the estimation of the model too, since the Range model can be estimated as a model with two different shift parameters in ascending/descending sequences (see Section 3.1).

All in all, based on Exxon Valdez data, the Range model: (1) confirms existing findings on the effect of respondent uncertainty; (2) offers an alternative explanation to anomalies in CV surveys.

\section{Conclusion}

In this article, we develop a model that allows us to deal with respondent uncertainty and starting-point bias in the same framework. This model is based on the principle of coherent arbitrariness, put forward by Ariely et al. (2003b). It allows for respondent uncertainty without having to rely on follow-up questions explicitly designed to measure the degree of that uncertainty (e.g., "How certain are you of your response?"). It provides an alternative interpretation of the fact the some of the responses to the second bid may be inconsistent with the responses to the first bid. This anomaly is explained by respondents' uncertainty, rather than anomalies in respondent behavior. Using the well-known Exxon Valdez survey, our empirical results suggest that, when uncertain, respondents tend to answer yes.

\footnotetext{
${ }^{7}$ DeShazo 2002, Cooper et al. 2002, Flachaire and Hollard 2006.
} 


\section{References}

Alberini, A., K. Boyle, and M. Welsh (2003). "Analysis of contingent valuation data with multiple bids and response options allowing respondents to express uncertainty". Journal of Environmental Economics and Management 45, 40-62.

Alberini, A., B. Kanninen, and R. Carson (1997). Modeling response incentive effects in dichotomous choice valuation data. Land Economics 73(3), 309-324.

Ariely, D., G. Loewenstein, and D. Prelec (2003a). Arbitrarily coherent preferences. In I. B. et J. Carillo (Ed.), The Psychology of Economic Decision. Oxford University Press.

Ariely, D., G. Loewenstein, and D. Prelec (2003b). "Coherent arbitrariness: Stable demand curves without stable preferences". Quarterly Journal of Economics 118-1, $73-105$.

Cameron, T. and J. Quiggin (1994). "Estimation using contingent valuation data from a dichotomous choice with follow-up questionnaire". Journal of Environmental Economics and Management 27, p218-234.

Carson, R., R. Mitchell, M. Hanemann, R. Kopp, S. Presser, and P. Ruud (2003). "Contingent valuation and lost passive use: Damages from the Exxon Valdez oil spill". Environmental and Resource Economics 25, 257-286.

Chapman, G. B. and E. J. Johnson (1999). "Anchoring, activation, and the construction of values". Organizational Behavior and Human Decision Processes 79(2), 115-153.

Cooper, J., W. M. Haneman, and G. Signorelli (2002). "One and one-half bids for contingent valuation". Review of Economics and Statistics 84, 742-750.

DeShazo, J. R. (2002). "Designing transactions without framing effects in iterative question formats". Journal of Environmental Economics and Management 43, p360-385.

Flachaire, E. and G. Hollard (2006). "Controlling starting-point bias in double-bounded contingent valuation surveys". Land Economics 82, 103-111.

Flachaire, E. and G. Hollard (2007). "Individual sensibility to framing effects in surveys". Journal of Economic Behavior and Organization, forthcoming.

Green, D., K. E. Kacowitz, D. Kahneman, and D. McFadden (1998). "Referendum contingent valuation, anchoring, and willingness to pay for public goods". Resource and Energy Economics 20, 85-116.

Hanemann, M. and B. Kanninen (1999). The statistical analysis of discrete response CV data. In I. Bateman and K. Willis (Eds.), Valuing Environmental Preferences. Theory and Practice of the Contingent Valuation Method in the US, EU, and Developing Countries, Chapter 11, pp. 302-441. New York: Oxford University Press.

Hanemann, W., J. Loomis, and B. Kanninen (1991). "Statistical efficiency of doublebounded dichotomous choice contingent valuation". American Journal of Agricultural Economics 73, 1255-1263.

Hanley, N. and B. Kriström (2002). "What's it worth? Exploring value uncertainty using interval questions in contingent valuation". working paper, 2002-10, University of Glasgow. 
Herriges, J. A. and J. F. Shogren (1996). "Starting point bias in dichotomous choice valuation with follow-up questioning". Journal of Environmental Economics and Management 30, 112-131.

Kanninen, B. (1995). "Bias in discrete response contingent valuation". Journal of Environmental Economics and Management 28, 114-25.

Li, C. Z. and L. Mattsson (1995). "Discrete choice under preference uncertainty: an improved structural model for contingent valuation". Journal of Environmental Economics and Management 28, 256-269.

Ready, R. C., S. Navrud, and W. R. Dubourg (2001). "How do respondents with uncertain willingness to pay answer contingent valuation questions?". Land Economics 77, 315-326.

Ready, R. C., J. C. Whitehead, and G. Blomquist (1995). "Contingent valuation when respondents are ambivalent". Journal of Environmental Economics and Management 29, 181-196.

Tversky, A. and D. Kahneman (1974). "Judgment under uncertainty: Heuristics and biases". Science 185, 124-131.

van Kooten, G. C., E. Krcmar, and E. H. Bulte (2001). "Preference uncertainty in non-market valuation: a fuzzy approach". American Journal of Agricultural Econmics 83, 487-500.

Welsh, M. P. and G. L. Poe (1998). "Elicitation effects in contingent valuation: comparisons to a multiple bounded discrete choice approach". Journal of Environmental Economics and Management 36, 170-185.

Whitehead, J. C. (2002). "Incentive incompatibility and starting-point bias in iterative valuation questions". Land Economics 78, p285-297. 